\title{
Las Competencias Profesionales y el Objeto en Diseño Industrial como Parte de la Formación del Diseñador en Cuba
}

\section{Professional Competences and Objects of Industrial Design as Part of the Designer Formation in Cuba}

\author{
M. Castellanos-Uralde' ${ }^{1}$ R. A. Ríos-Alonso ${ }^{1}$ \\ ${ }^{1}$ Instituto Superior de Diseño de Cuba (ISDi).
}

\section{RESUMEN}

La formación del diseñador a nivel universitario alcanza diversos niveles y teorías a escala internacional, y los modelos de enseñanza van desde el apego al arte hasta tendencias ingenieriles. En Cuba, la formación de los diseñadores parte de una estructura general: el genoma del diseñador, donde se definen esferas de actuación para cada carrera, y los modos de actuación de pregrado a posgrado. La formación actual se construye a partir del cumplimiento de objetivos, sin embargo, no se niega la veracidad de teorías contemporáneas, como la formación por competencias. Varias son las investigaciones en este tema que se han desarrollado en el marco del Instituto Superior de Diseño de Cuba (ISDi), declarando así las competencias necesarias para ambas ramas del diseño (comunicación visual e industrial) y muchas esferas declaradas en este genoma. El presente artículo muestra los resultados de la investigación correspondiente a las competencias profesionales a generar en el futuro diseñador de objetos en Cuba. La propuesta resultante es una solución estructural que define nueve competencias y tiene en cuenta la relación de conocimientos, habilidades y cualidades para la estructuración de la formación en la esfera objeto, lo que facilita su futura aplicación en programas formativos o de evaluación profesional a nivel laboral.

PALABRAS CLAVE: Objeto; competencias; habilidades; valores; formación.

\section{ABSTRACT}

Education of Designers in the university, follow different levels and theories internationally. Teaching models goes from arts to engineering tendencies in different ways. In Cuba, teaching theories becomes from a general structure: the designer genome, it declares: acting spheres for each career and acting ways depending the maturity and experience of designers. Actual formation becomes from declared objectives; anyway, it does not deny the validity of contemporary theories as the competence's one. Research from ISDi, declared Competences for both lines of Design and from many spheres declared in designer genome. This article shows the results of an investigation about the professional Competences for the Object sphere as first step in Industrial Design. Here we have a testimony of knowledge's, skills and values important for the Object Cuban designer in Cuba today. The resulting proposal is a structural solution that defines nine competences, which considers the relationship of knowledge, skills and qualities for the structuring of training in the subject area, which facilitates its future application in training programs or professional evaluation at work level.

KEYWORDS: Object; competences; skills, values, professional training.

Correspondencia:

DESTINATARIO: Marlen Castellanos Uralde INSTITUCIÓN: Instituto Superior de Diseño (ISDi) DIRECCIÓN: Belascoain núm. 710, entre Estrella y Maloja, Centro Habana, La Habana, C.P. 10300, Cuba. CORREO ELECTRÓNICO: marlencita2608@gmail.com

\section{Fecha de recepción:}

20 de junio de 2019

Fecha de aceptación:

3 de septiembre de 2019 


\section{INTRODUCCIÓN}

El perfeccionamiento de la educación en las diversas ramas ha sido fuente de interesantes resultados dentro de la investigación científica vinculada a la docencia. La forma de abordar los diferentes conocimientos, para lograr una formación óptima del profesional en Cuba, es un tema de constante búsqueda. Sin embargo, desde la formación por objetivos y capacidades, el mundo apunta hacia las competencias profesionales. No lejos de esto, el Instituto Superior de Diseño define las competencias requeridas para formar a sus profesionales en cada rama y esfera de actuación.

Dentro del Diseño Industrial, destaca la esfera objetual que es y será, por su escala y niveles de complejidad, el germen donde comienza dicha rama de la profesión. Es por ello que la presente investigación tiene como objetivo general identificar el sistema de competencias profesionales en la esfera de actuación: objeto, para el diseñador industrial.

El estudio del diseño de producto es el germen de donde parte toda indagación o trabajo del diseño industrial, es el núcleo de la profesión y eso lo hace inherente a la enseñanza del diseño desde sus inicios. La estructuración de esta enseñanza ha sido estudiada y modificada en función de las tendencias, los objetivos y el devenir del desarrollo científico-técnico. En Cuba $y$ en el mundo pudieran citarse muchos ejemplos de indagaciones sobre cómo y qué enseñar en cuanto al diseño de producto.

El enfoque de la formación cubana en diseño industrial presenta una marcada orientación hacia la ingeniería, pues se potencia en menos medida su vinculación con el arte, reconociendo que el diseño industrial no será de ninguna manera una ciencia exacta, sin embargo, está compuesto de elementos medibles y precisos en su vínculo con las tecnologías y las ciencias exactas. La producción industrial no estará aislada del conocimiento de los materiales y sus particularidades, de los procesos productivos, de la investigación e innovación tecnológica, de la mecanización, automatización y robótica, y para eso debe formarse al profesional del futuro. La academia brinda una serie de conocimientos básicos de diverso tipo que dota al profesional de los conocimientos fundamentales que le permitan desenvolverse en su profesión en diversas ramas y puestos de trabajo.
Entonces, la principal vinculación de los resultados mostrados, con la innovación tecnológica y la ingeniería, están dados por cuanto esta tipología de conocimientos repercute en la formación del profesional. Si bien, no resultan explícitos, sobre todo correspondientes a la ingeniería, se encuentran implícitos en los conocimientos y habilidades que se compilan en esta propuesta, los cuales tienen un corte eminentemente técnico y práctico, además de teórico.

Una vez definidos en la Academia Cubana de Diseño el Modelo de Gestión de las Competencias Profesionales del Diseño en Cuba ${ }^{[1]}$; Las Competencias Profesionales Específicas para Proyectar en el Diseño Industrial

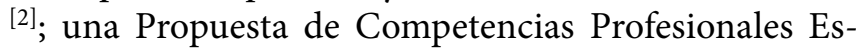
pecíficas Relacionadas con el Dominio de los Recursos Formales para el Diseño ${ }^{[3]}$; Elementos de Competencias a Desarrollar en Estudiantes de Diseño a través de la Enseñanza de los Materiales y Procesos ${ }^{[4]}$; las Competencias Profesionales Específicas del Diseñador para Desarrollar Proyectos de Diseño de Espacios Interiores ${ }^{[5]}$ y un Sistema de Competencias Tipográficas del Estudiante Cubano de Diseño ${ }^{[6]}$, resulta un vacío importante de conocimiento para la organización curricular y la estructuración de la docencia respecto al diseño industrial, la no definición de los elementos más significativos a desarrollar en la esfera básica del diseño industrial.

Si bien estudios similares han sido desarrollados en otros contextos, el adecuar esta propuesta a las características (atípicas) de la Cuba de hoy, se demuestra su validez, legitimada por la experimentación en la propia enseñanza del diseño de objetos industriales. La pluralidad de contextos alrededor del mundo particularizan, en última instancia, las características de esta propuesta para cada contexto, sin embargo, pueden anotarse una serie de conocimientos que son comunes para la enseñanza del diseño de producto a nivel internacional, lo cual puede ser, sin duda, caldo de cultivo para venideras investigaciones y artículos.

\section{METODOLOGÍA}

Para el abordaje del presente tema de investigación, se emplearon métodos del nivel teórico de la investigación científica, como son:

Histórico-lógico. Este método se utilizó para la indagación, estudio y determinación de los fundamentos teóricos relacionados con el devenir histórico de la forma- 
ción del diseñador en Cuba, así como de los estudios acerca de las competencias profesionales del diseñador y las competencias profesionales de la esfera de actuación: objeto.

Análisis y síntesis. Permitió profundizar en las tendencias acerca de las competencias profesionales del diseñador, arribar a regularidades y determinar las relaciones que se establecen entre las unidades y elementos como las partes que las conforman y su dinámica como un todo, así como el estudio de las características para construir el sistema de competencias profesionales para la esfera objeto en la formación del diseñador industrial.

Modelación. Se empleó para la abstracción mental de los elementos esenciales y las relaciones que se establecen en el sistema de competencias profesionales para la esfera de actuación: objeto, que se propone como vía de solución al problema de la investigación, con su correspondiente representación gráfica.

El enfoque sistémico. Con el objetivo de establecer las relaciones funcionales entre el sistema y los subsistemas que conforman la propuesta de competencias organizadas y articuladas, para la esfera de actuación objeto en la formación de los diseñadores industriales, permitió determinar su estructura, su desarrollo y el orden jerárquico de sus componentes.

Además, fueron seleccionados métodos del nivel empírico de la investigación científica como los siguientes:

Análisis documental. Se realizó para revisar los datos que aportaron los documentos del Plan de Estudios D, el Modelo del Profesional de Diseñador Industrial, los Programas de la Disciplina Principal Integradora de Diseño Industrial y de los Programas de Asignatura Diseño I y II, así como las encuestas de calidad del proceso docente, que proporcionaron elementos esenciales básicos para determinar las unidades y elementos de las competencias de la esfera de actuación: objeto, en la formación del diseñador industrial.

Entrevista a profesores. Se elaboró una guía de entrevista de forma grupal, con el objetivo de valorar sus criterios acerca de las habilidades que deben desarrollar los estudiantes en la esfera de actuación: objeto y su vínculo con las competencias.
Encuesta a estudiantes. Con el objetivo de valorar el criterio de los estudiantes sobre su preparación para el diseño en la esfera objeto, se aplicó un cuestionario a estudiantes de tercer año de diseño industrial durante los cursos 2015-2016 y de 2016-2017, aportando información para el diagnóstico del estado actual.

Consulta a especialistas. Se empleó con el objetivo de valorar los criterios acerca de la pertinencia del Sistema de Competencias Profesionales en la Esfera de Actuación: Objeto, como vía de solución al problema determinado. Se realizó a 6 especialistas con similares características en cuanto a experiencia como diseñadores en la esfera de actuación: objeto, como profesores del ISDi, con más de 10 años de trayectoria profesional.

La población seleccionada para el estudio de la problemática es de:

- 8 profesores que imparten las asignaturas Diseño Industrial durante tres cursos escolares y /o con las responsabilidades como Profesor Principal de Año, Jefe de Disciplina y Profesor Principal de la Asignatura.

- 100 Estudiantes del tercer y cuarto año de la carrera de Diseño Industrial de los cursos 2015 a 2017.

La muestra seleccionada para la investigación fue de 5 profesores con experiencia de trabajo, ya que los otros 3 son adiestrados con poca experiencia en la formación, además de 50 estudiantes de tercer año de la carrera de Diseño Industrial del curso 2015-2016.

La selección de los 5 especialistas para considerar sus opiniones y valorar la pertinencia del Sistema de Competencias Profesionales, se realizó bajo el criterio de que si bien son pocos los expertos con amplia práctica pedagógica en la esfera de actuación: objeto, tienen una vasta experiencia como diseñadores y formadores que se han vinculado con la evolución del diseño en Cuba.

\section{RESULTADOS Y DISCUSIÓN}

La investigación sobre las competencias en el modo de actuación: objeto, del diseñador industrial, se inició en el curso 2014-2015 y ha transitado del Plan de estudios $\mathrm{D}$ al Plan de estudios E, un momento de cambio, donde toda la estructura de la carrera, que funcionaba a partir del Plan D de la educación superior, cambia en función de una duración menor del tiempo de estudios (4 
años) y de nuevas transformaciones en la concepción del currículo.

Una invariante en la formación del diseñador industrial, como se señala, ha sido la proyección desde la carrera, con ejercicios encaminados a la solución de problemáticas económicas, tecnológicas y sociales, entre otras, abarcando el contexto social en cada momento histórico.

La necesidad de declaración de dichas competencias a nivel general y específico, parten de una organización en función de las esferas y modos de actuación profesional. Dicha propuesta parte además de la práctica pedagógica, con la necesidad de constatar problemas en la formación dentro de la esfera de actuación: ob- jeto, lo que se percibe en soluciones incorrectas o con escaso grado de perfección en los proyectos de diseño que realizan los estudiantes a lo largo de la carrera y específicamente en el tercer año académico (Plan D).

Para el desarrollo de dicha investigación, se tomó como muestra 5 profesores con experiencia de trabajo ( 3 años o más), ya que los otros 3 son adiestrados o instructores con poca experiencia en la formación, además de 50 estudiantes de tercer año de la carrera de Diseño Industrial del curso 2015-2016.

El Sistema de Competencias Profesionales para la Esfera de Actuación: Objeto consta a nivel cuantitativo, de la definición de 9 competencias, 15 unidades y 78 elementos de competencias (Tabla 1 y Apéndice-Tabla A).

TABLA 1.

Sistema de Competencias Profesionales para la Esfera de Actuación: Objeto

\begin{tabular}{|l|l|c|c|}
\hline \multicolumn{1}{|c|}{ CompETENCIAs } & $\begin{array}{c}\text { UNIDADES DE } \\
\text { COMPETENCIA }\end{array}$ & $\begin{array}{c}\text { ELEMENTOS DE } \\
\text { COMPETENCIA }\end{array}$ \\
\hline 1. & Conocimiento de la historia del Diseño Industrial. & 1 & 7 \\
\hline 2. & $\begin{array}{l}\text { Empleo de la organización metodológica del proceso de diseño establecida en el ISDi dirigida } \\
\text { a la esfera objeto. }\end{array}$ & 4 & 29 \\
\hline 3. & Estudio y determinación del uso de un objeto. & 2 & 8 \\
\hline 4. & Resolver las particularidades funcionales del objeto. & 1 & 6 \\
\hline 5. & Determinación de los aspectos tecnológicos y productivos del objeto. & 2 & 9 \\
\hline 6. & Adecuar el objeto a su contexto de interacción inmediato. & 1 & 2 \\
\hline 7. & Insertar el objeto en el mercado donde será comercializado. & 1 & 6 \\
\hline 8. & Poseer conocimientos sobre leyes y principios de la física. & 1 & 3 \\
\hline 9. & Dominar las técnicas de representación digital de objetos en 2D y 3D. & 2 & 7 \\
\hline
\end{tabular}

\begin{tabular}{|c|c|c|c|c|c|}
\hline Competencias & $\begin{array}{c}\text { UnidAdes DE } \\
\text { COMPETENCIA }\end{array}$ & $\begin{array}{c}\text { ElEMENTOS DE } \\
\text { COMPETENCIA }\end{array}$ & Conocimientos & Habilidades & VAlores \\
\hline 9 & 15 & 78 & 36 & 61 & 16 \\
\hline
\end{tabular}

La propuesta comienza respetando un orden cronológico, a partir del estudio de la Historia del Diseño Industrial, posteriormente se abordan las competencias a un nivel general, definiendo las relacionadas con la metodología del proceso de diseño: estructura medular en el abordaje de la carrera y que coincide con la formación en la esfera de actuación: objeto, y se cierra con competencias vinculadas a aspectos más específicos de la formación en la citada esfera de actuación.

Las competencias definidas son específicas, teniendo en cuenta su naturaleza profesional, toda vez que son transversales, por su inserción formativa en los perfiles profesionales del contexto nacional. Las presentes competencias profesionales logran precisar los conocimientos y habilidades a un nivel de precisión no al- canzado en el plano axiológico. Esta precisión se logra porque los valores transversales que se declaran y complementan la propuesta, son comunes para otras esferas de actuación y problemas profesionales.

Son resaltados de manera fática los valores a desarrollar en la formación del diseñador de objetos cubano en el siglo XXI (Apéndice-Tabla B), resaltando la creatividad, el pensamiento lógico, la actitud crítica y autocrítica, la capacidad argumentativa, la voluntad, la curiosidad, capacidad de experimentación, capacidad de trabajo en equipo, solidaridad, responsabilidad, motivación, receptividad, ética profesional, responsabilidad ecológica, humildad, humanismo y conciencia económica. 


\section{La propuesta en la aplicación cotidiana de sus resultados}

Toda la propuesta, desde el inicio de la investigación, funcionó como un laboratorio donde, de manera organizada, se fueron optimizando las propuestas de ejercicios prácticos a realizar, a partir de la coordinación horizontal de contenidos. Esta evolución fue dada a partir esta, más la vinculación interasignaturas, lo cual permitió constatar la importancia de las competencias aportadas por varias áreas del conocimiento tributando a la asignatura principal: Diseño Industrial (en este caso: I y II). La experiencia de un colectivo docente y la madurez desde el punto de vista del trabajo metodológico, permitieron organizar la experiencia y los resultados obtenidos con miras a la formación por competencias profesionales de los estudiantes del 3.er año de la carrera.

Es así como apreciamos la evolución de ejercicios como los ejercicios integradores de ambos semestres, que comenzaron siendo exclusivamente de Diseño Industrial y acabaron incorporando dos o más asignaturas con el paso del tiempo (Tabla 2).

TABLA 2.

Cursos del $1 .^{\circ}$ Y $2 .^{\circ}$ Semestres

\begin{tabular}{|c|c|c|c|}
\hline \multicolumn{4}{|c|}{ 1. ${ }^{\mathrm{ER}}$ SEMESTRE } \\
\hline Curso: 2014-2015 & CuRso: 2015-2016 & Curso: 2016-2017 & CuRso: 2017-2018 \\
\hline $\begin{array}{l}\text { TEC. No. 3: Se relaciona y tribu- } \\
\text { ta su evaluación solo a la asigna- } \\
\text { tura: Diseño Industrial I. }\end{array}$ & $\begin{array}{l}\text { TEC. No. 1: Se relaciona y tribu- } \\
\text { ta su evaluación a la asignatura: } \\
\text { Diseño Industrial I y a Tecnolo- } \\
\text { gía de los Metales. } \\
\text { TEC. No. 3: Se relaciona y tribu- } \\
\text { ta su evaluación a la asignatura: } \\
\text { Diseño Industrial I y a Tecnolo- } \\
\text { gía de los Plásticos. }\end{array}$ & $\begin{array}{l}\text { TEC. No. 3: Se relaciona y tribu- } \\
\text { ta su evaluación a la asignatura: } \\
\text { Diseño Industrial I, a Tecnolo- } \\
\text { gía de los Plásticos y Documen- } \\
\text { tación Técnica. }\end{array}$ & $\begin{array}{l}\text { TEC. No. 3: Se relaciona y tri- } \\
\text { buta su evaluación, a la asig- } \\
\text { natura: Diseño Industrial I, } \\
\text { a Tecnología de los Plásticos, } \\
\text { Computación III (Autodesk In- } \\
\text { ventor) y Documentación Téc- } \\
\text { nica. }\end{array}$ \\
\hline \multicolumn{4}{|l|}{ 2. ${ }^{\circ}$ SEMESTRE } \\
\hline $\begin{array}{l}\text { TEC. No. 3: Se relaciona y tri- } \\
\text { buta su evaluación a la asigna- } \\
\text { tura: Diseño Industrial II y a } \\
\text { Tecnología de la Madera. }\end{array}$ & $\begin{array}{l}\text { TEC. No. 2: Se relaciona y tribu- } \\
\text { ta su evaluación a la asignatura: } \\
\text { Diseño Industrial II, Tecnología } \\
\text { de Madera y a Metodología de la } \\
\text { Investigación. } \\
\text { TEC. No. 3: Se relaciona y tribu- } \\
\text { ta su evaluación a la asignatura: } \\
\text { Diseño Industrial II y a Historia } \\
\text { del Diseño II. }\end{array}$ & $\begin{array}{l}\text { TEC. No. 2: Se relaciona y tribu- } \\
\text { ta su evaluación a la asignatura: } \\
\text { Diseño Industrial II, Tecnología } \\
\text { de la Madera y a Metodología de } \\
\text { la Investigación. } \\
\text { TEC. No. 3: Se relaciona y tri- } \\
\text { buta su evaluación a la asignatu- } \\
\text { ra: Diseño Industrial II, Mode- } \\
\text { los y a Historia del Diseño II. }\end{array}$ & $\begin{array}{l}\text { TEC. No. 2: Se relaciona y tribu- } \\
\text { ta su evaluación a la asignatura: } \\
\text { Diseño Industrial II, Tecnología } \\
\text { de la Madera y a Metodología de } \\
\text { la Investigación. } \\
\text { TEC. No. 3: Se relaciona y tribu- } \\
\text { ta su evaluación a la asignatura: } \\
\text { Diseño Industrial II, Modelos y } \\
\text { a Historia del Diseño II. }\end{array}$ \\
\hline
\end{tabular}

De esta manera, se fueron agregando a las competencias relacionadas directamente al diseño de objeto detectadas desde el inicio de la investigación, una serie de competencias complementarias desde el nivel analítico, metodológico y tecnológico. Es de suponer que dichas competencias, se derivan directamente de los objetivos de las asignaturas y de cada uno de los ejercicios que cada una de ellas orienta (Apéndice-Figura $\mathrm{A}$ y Apéndice-Tabla C).

Los valores a desarrollar en el diseñador de objeto corresponden con los declarados en el modelo del profesional, los planes D y E y los programas de las diversas asignaturas vinculadas a esta esfera de actuación del diseño industrial.
Finalmente se comprobó en la práctica, el desarrollo de habilidades a partir de conocimientos impartidos y evaluados, conjuntamente con la formación de valores a partir de la sensibilización con problemas susceptibles de la sociedad, específicamente vinculadas a un grupo meta tan especial, como lo son los niños. Dentro de las conclusiones más importantes de esta experiencia, se encuentra el hecho de que estos valores a formar en los estudiantes, estos deben ser portados por los docentes. También resultó trascendental la vinculación a la realidad social y tecnológica del país y del ISDi en el desarrollo de las habilidades y en la formación de valores a partir de la ejecución de los ejercicios prácticos. La actitud frente al estudio, la conciencia ambiental, la capacidad de comunicación, la responsabilidad, solidaridad, capacidad de trabajo en equipo, la motivación y la humildad, 
fueron valores adquiridos o fomentados en los grupos de estudiantes, lo cual se comprueba en un alto grado de madurez y compromiso con el colectivo (incluidos estudiantes y profesores) (Apéndice-Figura B).

\section{CONCLUSIONES}

Una vez concluida la investigación para estructurar la propuesta del Sistema de Competencias en la Esfera: Objeto a formarse en el estudiante cubano de Diseño Industrial, y habiendo transitado todas las etapas planificadas para el cumplimiento del objetivo de la misma, se arriban a las siguientes conclusiones:

1. El estudio y manejo de la esfera de actuación objeto, es de gran importancia para el desempeño profesional del diseñador industrial. Esto implica que su formación debe delinearse de forma efectiva atendiendo a las necesidades del contexto en el que se inserta. En este sentido, se parte de una situación problemática caracterizada porque la práctica formativa hoy supera la declaración de los objetivos de la asignatura y la disciplina y no se encuentran declarados, de manera integral, todos los conocimientos y habilidades a desarrollar en el profesional de esta esfera.

La sistematización de basamentos teóricos, metodológicos y conceptuales, permiten elaborar una propuesta conceptual de competencias para la esfera de actuación: objeto como referencia para futuras investigaciones en este sentido.

2. El estudio realizado al contexto formativo que despliega el Instituto Superior de Diseño aporta resultados que demuestran la poca especificidad existente en los programas de estudio basados en objetivos. Además, evidencia que existen áreas del conocimiento que aún no alcanzan la calidad requerida en su formación. Los actores implicados en el proceso docente coinciden en la demanda de nuevas temáticas. Estos elementos permiten afirmar que existen condiciones favorables para la aplicación de una propuesta basada en competencias que haga más efectiva la asimilación y aplicación de los conocimientos en la esfera objeto.

3. La propuesta de competencias diseñada asume la base teórico-conceptual estudiada y la articula con los resultados del diagnóstico del contexto actual para arribar a una solución estructural que define $9 \mathrm{com}-$ petencias, desglosadas en 15 unidades y 78 elementos.
Su organización parte de la generación de varios grupos estructurados de manera ascendente en cuanto a complejidad y respetando un orden cronológico. La solución presentada tiene en cuenta la relación de conocimientos, habilidades y cualidades para la estructuración de la formación en la esfera objeto, lo que facilita su futura aplicación en programas formativos o de evaluación profesional a nivel laboral.

4. Los especialistas consultados para una valoración preliminar de la propuesta, coinciden en la evaluación positiva de la misma, ofreciendo criterios que convergen en la pertinencia de la solución, su validez como resultado científico y sus potencialidades de aplicación en el contexto formativo y empresarial cubano.

\section{REFERENCIAS}

[1] S. L. Peña, "Modelo de Gestión de las Competencias profesionales del Diseño en Cuba", Tesis de maestría, Instituto Superior de Diseño, La Habana, Cuba, 2007.

[2] J. Ramírez-Álvarez, Las competencias profesionales específicas para Proyectar en el Diseño Industrial. La Habana, Cuba: ISDi, 2011.

[3] E. Valle-Galindo, "Propuesta de Competencias Profesionales Específicas Relacionadas con el Dominio de los Recursos Formales para el Diseño", Tesis en opción al grado de Máster, Instituto Superior de Diseño, La Habana, Cuba, 2011.

[4] E. A. Navarro-Iglesias, "Elementos de Competencias a Desarrollar en Estudiantes de Diseño a Través de la Enseñanza de los Materiales y Procesos", Tesis en opción al Título de Máster, Instituto Superior de Diseño, 2014.

[5] M. I. Morales-Rey, Competencias Profesionales Especificas del Diseñador, para Desarrollar Proyectos de Diseño de Espacios Interiores. La Habana: ISDi, 2014.

[6] A. Aguilera-Torralbas, Sistema de Competencias Tipográficas del Estudiante Cubano de Diseño. La Habana: ISDi, 2017.

[7] Estrategia Ambiental Nacional, Ministerio de Ciencia, Tecnología y Medio Ambiente de Cuba, 2016 [en línea]. Disponible en: http://repositorio.geotech.cu/jspui/bitstream/1234/1511/6/Estrategia Ambiental Nacional 2016-2020.pdf [Acceso: 30 de junio de 2019]. 


\section{APÉNDICE}

TABLA A.

Propuesta del Sistema de Competencias Profesionales para la Esfera de Actuación: Objeto.

\begin{tabular}{|c|c|c|c|c|c|}
\hline $\begin{array}{l}\text { COMPETENCIAS } \\
\text { PROFESIONALES } \\
\text { ESPECÍFICAS }\end{array}$ & $\begin{array}{l}\text { UNIDADES DE } \\
\text { COMPETENCIAS }\end{array}$ & Elementos de Competencias & 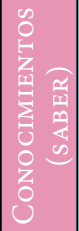 & 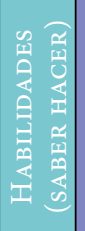 & 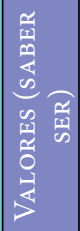 \\
\hline \multirow{7}{*}{$\begin{array}{l}\text { 1. Conocimiento de } \\
\text { la historia del dise- } \\
\text { ño industrial desde } \\
\text { su contexto. }\end{array}$} & \multirow{7}{*}{$\begin{array}{l}\text { 1.1. Dominar las } \\
\text { tendencias históri- } \\
\text { cas y estilos forma- } \\
\text { les del diseño en la } \\
\text { esfera objeto. }\end{array}$} & $\begin{array}{l}\text { 1.1.1. Conocer las características de los estilos formales y las tendencias his- } \\
\text { tóricas del diseño industrial. }\end{array}$ & & & \\
\hline & & $\begin{array}{l}\text { 1.1.2. Dominar los conceptos básicos y terminología técnica asociados al di- } \\
\text { seño de objetos industriales. }\end{array}$ & & & \\
\hline & & $\begin{array}{l}\text { 1.1.3. Conocer el desarrollo de los objetos industriales a través de la historia, } \\
\text { sus características y autores. }\end{array}$ & & & \\
\hline & & $\begin{array}{l}\text { 1.1.4. Dominar los principales exponentes nacionales e internacionales del } \\
\text { diseño industrial y su obra. }\end{array}$ & & & \\
\hline & & \begin{tabular}{|l|l} 
1.1.5. Analizar los referentes históricos en cuanto a los factores del diseño. \\
\end{tabular} & & & \\
\hline & & $\begin{array}{l}\text { 1.1.6. Conocer el desarrollo de la producción industrial y su vínculo con el } \\
\text { diseño. }\end{array}$ & & & \\
\hline & & $\begin{array}{l}\text { 1.1.7. Establecer referencias en estilos históricamente validados como punto } \\
\text { de partida para el diseño de nuevos objetos industriales. }\end{array}$ & & & \\
\hline \multirow{17}{*}{$\begin{array}{l}\text { 2. Empleo de la or- } \\
\text { ganización metodo- } \\
\text { lógica del proceso } \\
\text { de diseño estableci- } \\
\text { da en el ISDi dirigi- } \\
\text { da a la esfera objeto. }\end{array}$} & \multirow{6}{*}{$\begin{array}{l}\text { 2.1. Desarrollar el } \\
\text { trabajo con el en- } \\
\text { cargo de diseño. }\end{array}$} & 2.1.1. Conocer, describir y desarrollar la categoría: encargo de diseño. & & & \\
\hline & & $\begin{array}{l}\text { 2.1.2. Detectar las necesidades que pueden ser resueltas desde el diseño in- } \\
\text { dustrial. }\end{array}$ & & & \\
\hline & & $\begin{array}{l}\text { 2.1.3. Seleccionar y declarar condicionantes del proyecto a partir de un en- } \\
\text { cargo de diseño. }\end{array}$ & & & \\
\hline & & $\begin{array}{l}\text { 2.1.4. Dominar normas y regulaciones establecidas para la concepción e im- } \\
\text { plementación de cada objeto industrial (según sea el caso). }\end{array}$ & & & \\
\hline & & $\begin{array}{l}\text { 2.1.5. Definir las necesidades detectadas desde el punto de vista social, eco- } \\
\text { nómico y ecológico. }\end{array}$ & & & \\
\hline & & $\begin{array}{l}\text { 2.1.6. Comunicar adecuadamente los resultados de esta etapa de manera } \\
\text { oral, escrita y con la posibilidad del empleo de herramientas informáticas. }\end{array}$ & & & \\
\hline & \multirow[t]{6}{*}{$\begin{array}{l}\text { 2.2. Desarrollar la } \\
\text { etapa de problema. }\end{array}$} & $\begin{array}{l}\text { 2.2.1. Dominar herramientas y métodos a nivel teórico para el procesamien- } \\
\text { to de información. }\end{array}$ & & & \\
\hline & & $\begin{array}{l}\text { 2.2.2. Diferenciar y procesar los elementos pertenecientes al análisis de cada } \\
\text { uno de los factores de diseño como estudio previo a la concepción de un } \\
\text { objeto industrial. }\end{array}$ & & & \\
\hline & & $\begin{array}{l}\text { 2.2.3. Dominar el método de análisis-síntesis en el procesamiento de infor- } \\
\text { mación. }\end{array}$ & & & \\
\hline & & $\begin{array}{l}\text { 2.2.4. Compilar las conclusiones fundamentales dentro de cada análisis de } \\
\text { factores de diseño. }\end{array}$ & & & \\
\hline & & $\begin{array}{l}\text { 2.2.5. Seleccionar y expresar sintetizadamente, los elementos concluyentes } \\
\text { traducidos a requisitos de diseño. }\end{array}$ & & & \\
\hline & & $\begin{array}{l}\text { 2.2.6. Comunicar los resultados de la etapa, de manera oral y escrita, em- } \\
\text { pleando en los casos necesarios, herramientas informáticas. }\end{array}$ & & & \\
\hline & \multirow{5}{*}{$\begin{array}{l}\text { 2.3. Realización de } \\
\text { la etapa de concep- } \\
\text { tualización. }\end{array}$} & $\begin{array}{l}\text { 2.3.1. Correlacionar resultados de los análisis de factores de diseño para de- } \\
\text { finir las características principales del objeto. }\end{array}$ & & & \\
\hline & & $\begin{array}{l}\text { 2.3.2. Conocer y dominar los recursos básicos, básicos de relación, principios } \\
\text { y rangos de las cualidades formales propios del diseño básico de estructuras. }\end{array}$ & & & \\
\hline & & $\begin{array}{l}\text { 2.3.3. Expresar de manera oral y escrita, las categorías de la metodología } \\
\text { establecida en el ISDi para esta etapa: Premisas conceptuales, Alternativas } \\
\text { conceptuales, Alternativas de solución, Variantes y Concepto óptimo. }\end{array}$ & & & \\
\hline & & $\begin{array}{l}\text { 2.3.4. Seleccionar y evaluar las alternativas más pertinentes dentro de un } \\
\text { grupo de soluciones posibles. }\end{array}$ & & & \\
\hline & & $\begin{array}{l}\text { 2.3.5. Optimizar la forma, los elementos funcionales, de uso y tecnología, así } \\
\text { como la adecuación al mercado y contexto de uso del objeto. }\end{array}$ & & & \\
\hline
\end{tabular}




\begin{tabular}{|c|c|c|c|c|c|}
\hline $\begin{array}{l}\text { COMPETENCIAS } \\
\text { PROFESIONALES } \\
\text { ESPECÍFICAS }\end{array}$ & $\begin{array}{l}\text { UNIDADES DE } \\
\text { COMPETENCIAS }\end{array}$ & Elementos de Competencias & 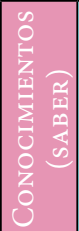 & 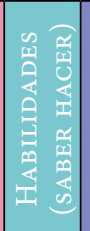 & 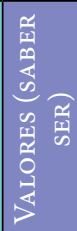 \\
\hline & & $\begin{array}{l}\text { 2.3.6. Emplear las características de estilos y tendencias históricas en el pro- } \\
\text { ceso de conceptualización. }\end{array}$ & & & \\
\hline & & $\begin{array}{l}\text { 2.3.7. Expresar de manera verbal (oral y escrita) las características (funda- } \\
\text { mentales y generales) del objeto diseñado. }\end{array}$ & & & \\
\hline & & $\begin{array}{l}\text { 2.3.8. Emplear la forma como generador/representador de conceptos de di- } \\
\text { seño. }\end{array}$ & & & \\
\hline & & $\begin{array}{l}\text { 2.3.9. Representar gráficamente (manual o digitalmente), la solución formal } \\
\text { del objeto. }\end{array}$ & & & \\
\hline & & $\begin{array}{l}\text { 2.3.10. Reconocer las tipologías de proyectos de diseño de objeto según sus } \\
\text { complejidades (objeto y sistema de productos). }\end{array}$ & & & \\
\hline & & $\begin{array}{l}\text { 2.3.11. Conocer los elementos que pautan la unidad entre los componentes } \\
\text { de un sistema de productos del diseño industrial. }\end{array}$ & & & \\
\hline & & $\begin{array}{l}\text { 2.3.12. Comunicar con alto grado de eficiencia y precisión, las características } \\
\text { y detalles de la solución final. }\end{array}$ & & & \\
\hline & $\begin{array}{l}\text { 2.4. Ejecución de la } \\
\text { etapa de Desarrollo. }\end{array}$ & $\begin{array}{l}\text { 2.4.1. Dominar la construcción de maquetas y modelos representativos a es- } \\
\text { cala. }\end{array}$ & & & \\
\hline & & $\begin{array}{l}\text { 2.4.2. Dominar y emplear las normas vigentes para la representación técnica } \\
\text { de los elementos que tributan a la producción industrial. }\end{array}$ & & & \\
\hline & & $\begin{array}{l}\text { 2.4.3. Representar los ajustes de la solución a la producción industrial, con } \\
\text { alto nivel de precisión, empleando los estilos y normas estipuladas. }\end{array}$ & & & \\
\hline & & $\begin{array}{l}\text { 2.4.4. Adecuar el objeto al lenguaje de una tecnología, producción e indus- } \\
\text { tria específica. }\end{array}$ & & & \\
\hline & & $\begin{array}{l}\text { 2.4.5. Comunicar a los especialistas, de manera oral, gráfica y escrita, los } \\
\text { ajustes de la solución, con el empleo de los términos técnicos de la profesión } \\
\text { y la industria. }\end{array}$ & & & \\
\hline \multirow{9}{*}{$\begin{array}{l}\text { 3. Estudio y deter- } \\
\text { minación del uso } \\
\text { de un objeto }\end{array}$} & \multirow{5}{*}{$\begin{array}{l}\text { 3.1. Resolver ana- } \\
\text { líticamente, el uso } \\
\text { del objeto indus- } \\
\text { trial a diseñar. }\end{array}$} & $\begin{array}{l}\text { 3.1.1. Analizar similares diacrónicos y sincrónicos para determinar posibles } \\
\text { modos, secuencias y esquemas de uso. }\end{array}$ & & & \\
\hline & & 3.1.2. Definir los elementos que interfieren en el uso. & & & \\
\hline & & $\begin{array}{l}\text { 3.1.3. Representar esquemáticamente la configuración funcional del posible } \\
\text { resultado. }\end{array}$ & & & \\
\hline & & $\begin{array}{l}\text { 3.1.4. Determinar el modo, secuencia, intensidad y frecuencia de uso (de ser } \\
\text { pertinente). }\end{array}$ & & & \\
\hline & & $\begin{array}{l}\text { 3.1.5. Arribar a conclusiones, determinando elementos indispensables con } \\
\text { respecto al uso. }\end{array}$ & & & \\
\hline & \multirow{4}{*}{$\begin{array}{l}3.2 . \text { Determinar } \\
\text { los elementos re- } \\
\text { lacionados con la } \\
\text { interacción física } \\
\text { entre el usuario y el } \\
\text { objeto. }\end{array}$} & $\begin{array}{l}\text { 3.2.1. Aplicar métodos y normas de la Ergonomía para el análisis y la toma } \\
\text { de decisiones en cuanto a dimensionamientos, usabilidad y lógica de uso. }\end{array}$ & & & \\
\hline & & $\begin{array}{l}\text { 3.2.2. Seleccionar y distribuir con alto grado de coherencia formal, las textu- } \\
\text { ras, colores y materiales a emplear. }\end{array}$ & & & \\
\hline & & $\begin{array}{l}\text { 3.2.3. Describir de manera oral y escrita, las características, preferencias, } \\
\text { cualidades y deficiencias del público meta al cual se dirigirá finalmente el } \\
\text { objeto. }\end{array}$ & & & \\
\hline & & $\begin{array}{l}\text { 3.2.4. Analizar y seleccionar las particularidades principales de los usuarios } \\
\text { meta para ajustar el producto en cada caso. }\end{array}$ & & & \\
\hline \multirow{6}{*}{$\begin{array}{l}\text { 4. Resolver las par- } \\
\text { ticularidades fun- } \\
\text { cionales del objeto. }\end{array}$} & \multirow{6}{*}{$\begin{array}{l}\text { 4.1. Determinar los } \\
\text { elementos funcio- } \\
\text { nales del objeto. }\end{array}$} & $\begin{array}{l}\text { 4.1.1. Dominar a nivel conceptual y práctico, el funcionamiento de los obje- } \\
\text { tos industriales general y específicamente. }\end{array}$ & & & \\
\hline & & 4.1.2. Seleccionar principios de funcionamiento pertinentes. & & & \\
\hline & & $\begin{array}{l}\text { 4.1.3. Seleccionar los portadores funcionales que posibiliten una interacción } \\
\text { adecuada con el objeto. }\end{array}$ & & & \\
\hline & & $\begin{array}{l}\text { 4.1.4. Reconocer los elementos, partes y piezas que influyen en el funciona- } \\
\text { miento de un objeto. }\end{array}$ & & & \\
\hline & & $\begin{array}{l}\text { 4.1.5. Proponer la distribución funcional interna de las partes y componen- } \\
\text { tes. }\end{array}$ & & & \\
\hline & & 4.1.6. Adecuar a la reparación y/o sustitución de partes y piezas del objeto. & & & \\
\hline
\end{tabular}




\begin{tabular}{|c|c|c|c|c|c|}
\hline $\begin{array}{l}\text { COMPETENCIAS } \\
\text { PROFESIONALES } \\
\text { ESPECÍFICAS }\end{array}$ & $\begin{array}{l}\text { UNIDADES DE } \\
\text { COMPETENCIAS }\end{array}$ & ELEMENTOS DE COMPETENCIAS & 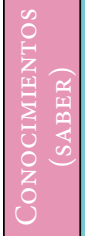 & 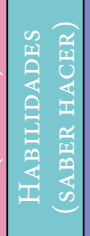 & 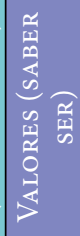 \\
\hline \multirow{9}{*}{$\begin{array}{l}\text { 5. Determinar los } \\
\text { aspectos tecnológi- } \\
\text { cos y productivos } \\
\text { del objeto. }\end{array}$} & \multirow{4}{*}{$\begin{array}{l}\text { 5.1. Poseer cono- } \\
\text { cimientos respecto } \\
\text { a las tecnologías } \\
\text { y materiales en la } \\
\text { producción indus- } \\
\text { trial. }\end{array}$} & $\begin{array}{l}\text { 5.1.1. Dominar y analizar los procesos de producción industrial en Cuba } \\
\text { (dentro y fuera del ISDi). }\end{array}$ & & & \\
\hline & & $\begin{array}{l}\text { 5.1.2. Dominar los ajustes declarados en el proceso de Diseño para poner a } \\
\text { punto la producción industrial. }\end{array}$ & & & \\
\hline & & $\begin{array}{l}\text { 5.1.3. Dominar las propiedades estructurales y físicas de los posibles mate- } \\
\text { riales a emplear en la producción. }\end{array}$ & & & \\
\hline & & $\begin{array}{l}\text { 5.1.4. Seleccionar las tecnologías y materiales óptimos para la producción } \\
\text { industrial (en cada caso). }\end{array}$ & & & \\
\hline & \multirow{5}{*}{$\begin{array}{l}\text { 5.2. Conocer, } \\
\text { respetar términos } \\
\text { ambientales con } \\
\text { respecto a la con- } \\
\text { cepción del ciclo de } \\
\text { vida del objeto (Es- } \\
\text { trategia Ambiental } \\
\text { Nacional) }\end{array}$} & $\begin{array}{l}\text { 5.2.1. Conocer el origen de los materiales y características de la tecnología } \\
\text { a emplear. }\end{array}$ & & & \\
\hline & & $\begin{array}{l}\text { 5.2.2. Descripción y selección de elementos determinantes en la situación } \\
\text { ambiental que rodea el ciclo de vida del objeto. }\end{array}$ & & & \\
\hline & & $\begin{array}{l}\text { 5.2.3. Estudiar y concebir de la vida útil del producto conjuntamente con su } \\
\text { posible huella ecológica. }\end{array}$ & & & \\
\hline & & $\begin{array}{l}\text { 5.2.4. Selección de mejoras ecológicas y/o medioambientales posibles a im- } \\
\text { plementar en el diseño final. }\end{array}$ & & & \\
\hline & & 5.2.5. Analizar y resolver el ciclo de vida del objeto. & & & \\
\hline \multirow{2}{*}{$\begin{array}{l}\text { 6. Adecuar el obje- } \\
\text { to a su contexto de } \\
\text { interacción inme- } \\
\text { diato. }\end{array}$} & \multirow{2}{*}{$\begin{array}{l}\text { 6.1. Estudio del } \\
\text { contexto de uso in- } \\
\text { mediato. }\end{array}$} & $\begin{array}{l}\text { 6.1.1. Describir y analizar las características del medio que rodeará al objeto } \\
\text { en su uso (condiciones de iluminación, humedad, actividades paralelas, con- } \\
\text { vivencia, cromas, acabados, materiales). }\end{array}$ & & & \\
\hline & & $\begin{array}{l}\text { 6.1.2. Tomar decisiones con respecto a la adecuación el objeto al contexto } \\
\text { de uso. }\end{array}$ & & & \\
\hline \multirow{6}{*}{$\begin{array}{l}\text { 7. Insertar el objeto } \\
\text { en el mercado don- } \\
\text { de será comerciali- } \\
\text { zado. }\end{array}$} & \multirow{6}{*}{$\begin{array}{l}\text { 7.1. Conocimiento } \\
\text { del mercado y sus } \\
\text { características. }\end{array}$} & $\begin{array}{l}\text { 7.1.1. Detectar posibles nichos de mercado donde insertar el nuevo produc- } \\
\text { to. }\end{array}$ & & & \\
\hline & & 7.1.2. Adecuar la solución al futuro almacenamiento y traslado. & & & \\
\hline & & $\begin{array}{l}\text { 7.1.3. Conocer y respetar las normas de almacenamiento y transporte de } \\
\text { cada objeto. }\end{array}$ & & & \\
\hline & & $\begin{array}{l}\text { 7.1.4. Conocer las tecnologías, dimensiones, y características de los envases } \\
\text { y embalajes que almacenarán y permitirán la transportación del objeto di- } \\
\text { señado. }\end{array}$ & & & \\
\hline & & $\begin{array}{l}\text { 7.1.5. Conocer, analizar y resumir de las características principales de la } \\
\text { competencia. }\end{array}$ & & & \\
\hline & & $\begin{array}{l}\text { 7.1.6. Adecuar la solución a las normas, características y competencia de- } \\
\text { claradas. }\end{array}$ & & & \\
\hline \multirow{3}{*}{$\begin{array}{l}\text { 8. Poseer conoci- } \\
\text { mientos sobre leyes } \\
\text { y principios de la } \\
\text { física. }\end{array}$} & \multirow{3}{*}{$\begin{array}{l}\text { 8.1. Dominar las le- } \\
\text { yes de la física que } \\
\text { influyen en el fun- } \\
\text { cionamiento de los } \\
\text { objetos. }\end{array}$} & $\begin{array}{l}\text { 8.1.1. Conocer acerca de la resistencia y características físicas de los mate- } \\
\text { riales. }\end{array}$ & & & \\
\hline & & $\begin{array}{l}\text { 8.1.2. Dominar y emplear las leyes de la naturaleza, que permiten el funcio- } \\
\text { namiento de los objetos. }\end{array}$ & & & \\
\hline & & $\begin{array}{l}\text { 8.1.3. Aplicación práctica de los cálculos correspondientes a cada una de } \\
\text { estas leyes y principios. }\end{array}$ & & & \\
\hline \multirow{6}{*}{$\begin{array}{l}\text { 9. Dominar las téc- } \\
\text { nicas de representa- } \\
\text { ción digital de obje- } \\
\text { tos en } 2 \mathrm{D} \text { y } 3 \mathrm{D} \text {. }\end{array}$} & \multirow{5}{*}{$\begin{array}{l}\text { 9.1. Dominio de las } \\
\text { técnicas de repre- } \\
\text { sentación } 2 \mathrm{D} \text {. }\end{array}$} & 9.1.1. Dominar y emplear el dibujo en perspectiva e isométrico. & & & \\
\hline & & 9.1.2. Dominar la escala en la representación. & & & \\
\hline & & 9.1.3. Dominar el color y las técnicas para aplicarlo. & & & \\
\hline & & $\begin{array}{l}\text { 9.1.4. Dominar software (vectoriales o no) para el trabajo de imágenes bidi- } \\
\text { mensionales y planos técnicos. }\end{array}$ & & & \\
\hline & & $\begin{array}{l}\text { 9.2.1. Dominar y emplear software que permitan la simulación digital del } \\
\text { objeto en } 3 \mathrm{D} \text { y sus detalles. }\end{array}$ & & & \\
\hline & $\begin{array}{l}\text { 9.2. Dominio de las } \\
\text { técnicas de repre- } \\
\text { sentación 3D. }\end{array}$ & \begin{tabular}{|l|} 
9.2.2. Conocer las nuevas tecnologías de impresión 3D. \\
9.2.3. Dominar y emplear software que posibiliten la representación de ma- \\
teriales y detalles del objeto con alto grado de fidelidad.
\end{tabular} & & & \\
\hline
\end{tabular}


TABLA B

Valores que han de Presentarse en el Desarrollo de todas las Competencias del Diseñador Cubano en la Esfera de ACTUACIÓN: ОвJETO

\begin{tabular}{|c|l|}
\hline NúM. & \multicolumn{1}{|c|}{ VALORES } \\
\hline 1 & Creatividad \\
\hline 2 & Pensamiento lógico \\
\hline 3 & Capacidad argumentativa \\
\hline 4 & Voluntad \\
\hline 5 & Curiosidad \\
\hline 6 & Capacidad de experimentación \\
\hline 7 & Capacidad de trabajo en equipo \\
\hline 8 & Actitud crítica y autocrítica \\
\hline 9 & Solidaridad \\
\hline 10 & Responsabilidad \\
\hline 11 & Motivación \\
\hline 12 & Receptividad \\
\hline 13 & Ética profesional \\
\hline 14 & Responsabilidad ecológica \\
\hline 15 & Humildad \\
\hline 16 & Humanismo \\
\hline 17 & Responsabilidad económica \\
\hline
\end{tabular}

TABLA C.

Vinculación de la Propuesta con las Categorías de los Documentos Rectores de la Carrera

\begin{tabular}{|c|c|}
\hline $\begin{array}{l}\text { COMPETENCIAS } \\
\text { PROFESIONALES } \\
\text { ESPECÍFICAS } \\
\end{array}$ & $\begin{array}{c}\text { Elemento del Modelo } \\
\text { DEL ProfesionAl AL QUE } \\
\text { SE ASOCIA }\end{array}$ \\
\hline $\begin{array}{l}\text { 1. Conocimiento de la histo- } \\
\text { ria del Diseño Industrial. }\end{array}$ & $\begin{array}{l}\text { - Objetivos del año } \\
\text { - Esfera de actuación: objeto } \\
\text { - Modo de actuación: investigar } \\
\text { - Factores de diseño }\end{array}$ \\
\hline $\begin{array}{l}\text { 2. Empleo de la organización } \\
\text { metodológica del proceso de } \\
\text { diseño establecida en el ISDi } \\
\text { dirigida a la esfera: objeto. }\end{array}$ & $\begin{array}{l}\text { - Metodología del proceso de diseño } \\
\text { - Objetivos generales instructivos } \\
\text { - Modo de actuación: proyectar } \\
\text { - Esfera de actuación: objeto } \\
\text { - Objetivos del año }\end{array}$ \\
\hline $\begin{array}{l}\text { 3. Estudio y determinación } \\
\text { del uso de un objeto. }\end{array}$ & $\begin{array}{l}\text { - Factores de diseño } \\
\text { - Esfera de actuación: objeto } \\
\text { - Objetivos del año }\end{array}$ \\
\hline $\begin{array}{l}\text { 4. Resolver las funciones y el } \\
\text { funcionamiento de los obje- } \\
\text { tos industriales. Resolver las } \\
\text { particularidades funcionales } \\
\text { del objeto. }\end{array}$ & $\begin{array}{l}\text { - Factores de diseño } \\
\text { - Esfera de actuación: objeto } \\
\text { - Objetivos del año }\end{array}$ \\
\hline $\begin{array}{l}\text { 5. Determinación de los } \\
\text { aspectos tecnológicos y } \\
\text { productivos del objeto. }\end{array}$ & $\begin{array}{l}\text { - Factores de diseño } \\
\text { - Esfera de actuación: objeto } \\
\text { - Objetivos del año } \\
\end{array}$ \\
\hline $\begin{array}{l}\text { 6. Adecuar el objeto a su } \\
\text { contexto de interacción } \\
\text { inmediato. }\end{array}$ & $\begin{array}{l}\text { - Factores de diseño } \\
\text { - Esfera de actuación: objeto } \\
\text { - Objetivos del año } \\
\end{array}$ \\
\hline $\begin{array}{l}\text { 7. Insertar el objeto en el } \\
\text { mercado donde será comer- } \\
\text { cializado. }\end{array}$ & $\begin{array}{l}\text { - Factores de diseño } \\
\text { - Esfera de actuación: objeto } \\
\text { - Objetivos del año } \\
\end{array}$ \\
\hline $\begin{array}{l}\text { 8. Poseer conocimientos } \\
\text { sobre leyes y principios de } \\
\text { la física. }\end{array}$ & $\begin{array}{l}\text { - Modo de actuación: proyectar } \\
\text { - Esfera de actuación: objeto } \\
\text { - Objetivos del año }\end{array}$ \\
\hline $\begin{array}{l}\text { 9. Dominar las técnicas de } \\
\text { representación digital de } \\
\text { objetos en } 2 \mathrm{D} \text { y } 3 \mathrm{D} \text {. }\end{array}$ & $\begin{array}{l}\text { - Modo de actuación: proyectar } \\
\text { - Objetivos del año }\end{array}$ \\
\hline
\end{tabular}

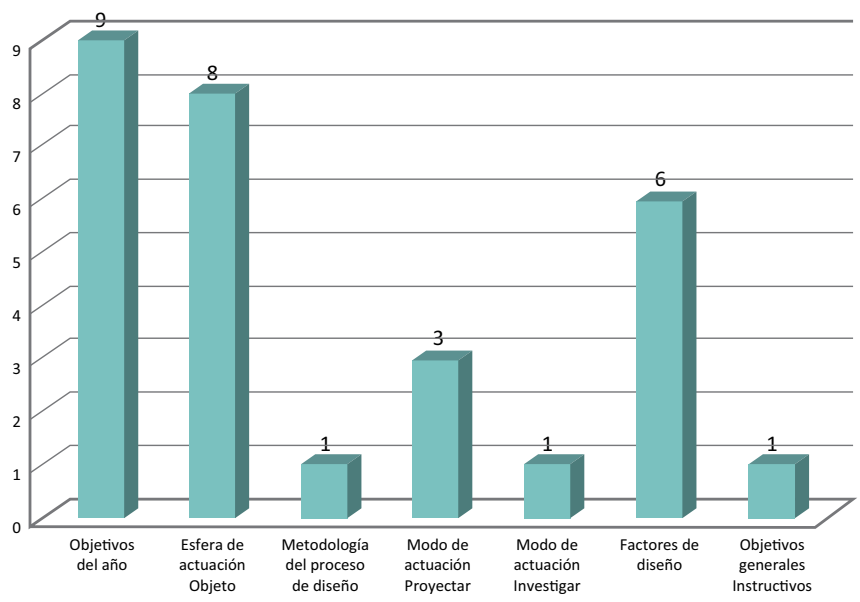

Figura A. Categorías del modelo del profesional, vinculadas a las competencias profesionales declaradas.

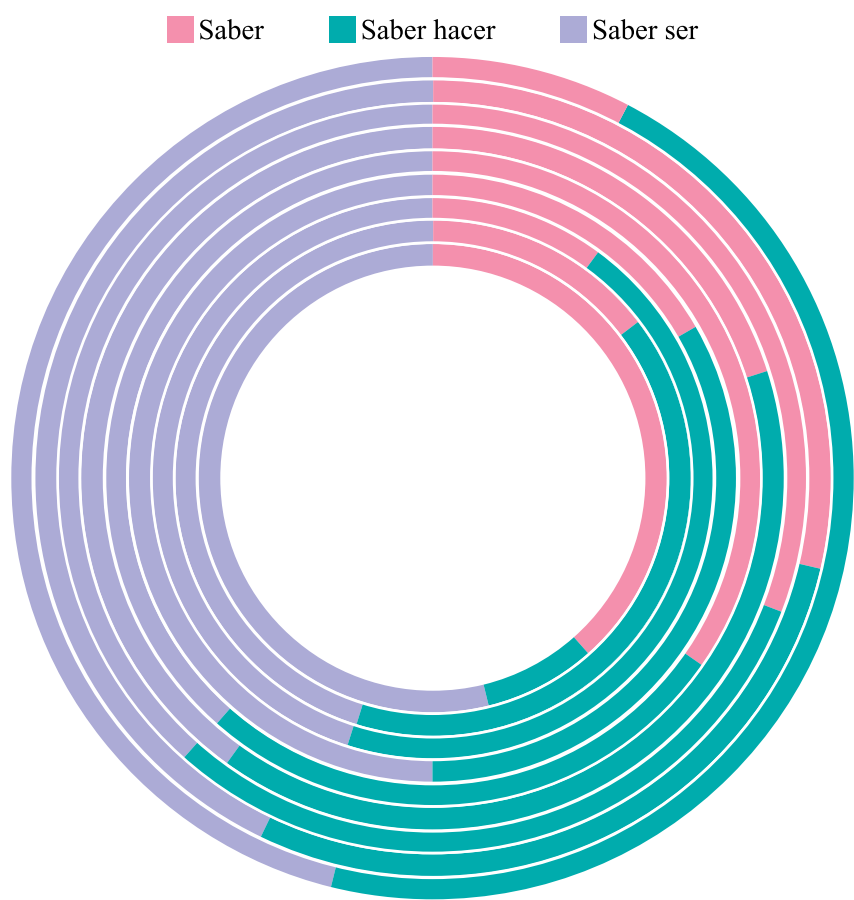

Figura B. Representación de los conocimientos, habilidades y capacidades en cada competencia declarada. 